\title{
Stress tensor and gradient of hydrostatic pressure in the contact plane of axisymmetric bodies under normal and tangential loading
}

\author{
Emanuel Willert $^{1}$ (i) | Fabian Forsbach $^{1}$ | Valentin L. Popov ${ }^{1,2}$
}

${ }^{1}$ Technische Universität Berlin, Berlin, 10623 Germany

${ }^{2}$ National Research Tomsk State University, Tomsk, 634050, Russia

Correspondence

Emanuel Willert, Technische Universität Berlin, 10623, Berlin, Germany

Email:e.willert@tu-berlin.de
The Hertzian contact theory, as well as most of the other classical theories of normal and tangential contact, provides displacements and the distribution of normal and tangential stress components directly in the contact surface. However, other components of the full stress tensor in the material may essentially influence the material behaviour in contact. Of particular interest are principal stresses and the equivalent von Mises stress, as well as the gradient of the hydrostatic pressure. For many engineering and biomechanical problems, it would be important to find these stress characteristics at least in the contact plane. In the present paper, we show that the complete stress state in the contact plane can be easily found for axially symmetric contacts under very general assumptions. We provide simple explicit equations for all stress components and the normal component of the gradient of hydrostatic pressure in the form of onedimensional integrals.

\section{K E Y W O R D S}

axial symmetry, friction, method of dimensionality reduction, normal contact, pressure gradient, stress state, tangential contact

\section{1 | INTRODUCTION}

Solutions of contact problems should provide interrelations between applied macroscopic forces and resulting macroscopic displacements of contacting bodies, as well as their stress state. However, the "stress state" is in most cases limited to the normal stress component (which is typically called "pressure") and one (or, in some circumstances, two) tangential in-plane stress components. Theoretically, the knowledge of the stress components in the contact surface is sufficient for calculating the complete stress tensor in the whole volume of the contacting bodies. To this sake, one could utilize the fundamental solutions by Boussinesq [1] and Cerruti [2] and use the superposition principle. Alternatively it is possible to apply the solution in terms of elastic potentials given by Barber [3] (Appendix A). However, this theoretical possibility is rarely realized practically. The reason is, that explicit closed-form solutions for the stresses inside the contacting bodies are scarce and usually related to the Hertzian contact [4] - see the works by Huber [5] and Sackfield \& Hills [6,7] on normal loading and the publications by Hamilton \& Goodman [8, 9] and Sackfield \& Hills [10] on tangential loading.

In the general case, the stresses have to be calculated numerically which requires the evaluation of integrals with computational complexity of the order $N^{5}$, where $N$ is the number of elements in one direction of the contact plane. A "compromise" approach

This is an open access article under the terms of the Creative Commons Attribution License, which permits use, distribution and reproduction in any medium, provided the original work is properly cited. 
could be to consider the complete stress tensor directly in the contact plane. Such information would allow a better qualitative analysis of the stress state and its influence on mechanical behaviour of the materials. In particular, the maximum negative principal stress could indicate the positions of possible initiation of fatigue cracks. Moreover, the gradient of the hydrostatic pressure could provide indications for intensity of mechanically induced diffusion (or in biological systems, like natural joints, the intensity of "pumping" of the filling fluid - which is an essential process determining the growth of tissue). However, determination of the stresses in the contact plane by direct integration is still a numerical operation of complexity $N^{4}$.

Of course, above mentioned explicit solutions [5,9], contain the stress state in the surface as a limiting case for $z=0$ (note, that we don't just refer to the traction vector at the surface, but the full stress state). If the solution is in closed form, this limiting procedure can be done without difficulties. However, these solutions are limited only to special geometries. In the general case, no simple expressions for the stress state and related quantities in the contact plane can be found in the literature. In the present paper, we will show that, in the case of axisymmetric contacts, all components of the stress tensor, as well as the pressure gradient, can be given in a simple integral form, which can be used for both analytical solutions and numerical simulations. The derivation is based on a superposition idea, which stems from Mossakovski [11] and which is detailed in the following section. Note, that an alternative but theoretically and computationally more complex approach for determining the stresses inside the bodies in axisymmetric contact problems is also the correspondence between axisymmetric and plane contact problems, as demonstrated by Heß [12] (Chapter 2.4).

The present manuscript is organized as follows: First we will derive simple expressions for the stress state and the gradient of hydrostatic pressure in the contact plane for axisymmetric normal and tangential contacts within the Hertz-Mindlin approximations. This is done based on an appropriate superposition of respective solutions for the cylindrical flat punch problem. After that, in Section 4, the conical contact is studied in detail as an illustrative example for the general expressions given before, including stress components, the von Mises equivalent stress and the gradient of hydrostatic pressure. A discussion and some conclusive remarks close the manuscript.

\section{I FRICTIONLESS NORMAL CONTACT}

In this section, we give simple analytic expressions for the stress state and the pressure gradient in the contact plane for the axisymmetric Boussinesq problem. As first suggested by Mossakovski [11], the solution for an arbitrary axisymmetric profile can be obtained, if the respective solution of a contact with a flat-ended cylindrical punch is known. We thus first require the respective solutions for the indentation of an elastic half-space by a rigid cylindrical flat punch. By superimposing incremental flat punch solutions, we then can easily construct the general axisymmetric solution.

\section{1 | The flat punch solution}

As stated above, we require the solution for the desired stress components and the pressure gradient in case of indentation by a flat cylindrical punch, to obtain the superposition expressions for arbitrary axisymmetric indenter profiles. For convenience, we will start with the known results for the Hertzian contact, to derive the respective flat punch solution.

According to Huber [5], the non-vanishing stress components in the contact plane $z=0$ for the frictionless indentation of an elastic half-space by a parabolic rigid body with the radius of curvature $R$ in the vicinity of the contact in physical polar coordinates $\{r, \varphi, z\}$ are given by the expressions

$$
\begin{aligned}
& \sigma_{r r}^{\mathrm{H}}(r>a ; a)=-\sigma_{\varphi \varphi}^{\mathrm{H}}(r>a ; a)=\frac{2 E^{*} a}{\pi R} \frac{1-2 v}{3} \frac{a^{2}}{r^{2}}, \\
& \sigma_{r r}^{\mathrm{H}}(r \leq a ; a)=\frac{2 E^{*} a}{\pi R}\left\{\frac{1-2 v}{3} \frac{a^{2}}{r^{2}}\left[1-\left(\frac{\sqrt{a^{2}-r^{2}}}{a}\right)^{3}\right]-\frac{\sqrt{a^{2}-r^{2}}}{a}\right\}, \\
& \sigma_{\varphi \varphi}^{\mathrm{H}}(r \leq a ; a)=-\frac{2 E^{*} a}{\pi R}\left\{\frac{1-2 v}{3} \frac{a^{2}}{r^{2}}\left[1-\left(\frac{\sqrt{a^{2}-r^{2}}}{a}\right)^{3}\right]+\frac{2 v \sqrt{a^{2}-r^{2}}}{a}\right\}, \\
& \sigma_{z z}^{\mathrm{H}}(r \leq a ; a)=-\frac{2 E^{*} a}{\pi R} \frac{\sqrt{a^{2}-r^{2}}}{a} .
\end{aligned}
$$


Here $E^{*}=E /\left(1-v^{2}\right)$ is the effective Young's modulus and $v$ Poisson ratio, and $a$ denotes the contact radius. The last expression is, of course, simply the Hertzian "pressure" distribution. Due to the superposition idea, the respective solutions for the indentation by a rigid cylindrical flat punch with the radius $a$ can be calculated from above expressions via

$$
\sigma_{i j}^{\mathrm{FP}}=\delta \frac{\partial \sigma_{i j}^{\mathrm{H}}}{\partial \delta^{\mathrm{H}}}=\delta \frac{\partial \sigma_{i j}^{\mathrm{H}}}{\partial a} \frac{\mathrm{d} a}{\mathrm{~d} \delta^{\mathrm{H}}}=\delta \frac{\partial \sigma_{i j}^{\mathrm{H}}}{\partial a} \frac{R}{2 a},
$$

with the indentation depth $\delta$ and $\delta^{\mathrm{H}}=a^{2} / R$ in the Hertzian case. Hence,

$$
\begin{aligned}
& \sigma_{r r}^{\mathrm{FP}}(r>a ; a)=-\sigma_{\varphi \varphi}^{\mathrm{FP}}(r>a, z=0 ; a)=\frac{E^{*} \delta(1-2 v) a}{\pi r^{2}}, \\
& \sigma_{r r}^{\mathrm{FP}}(r \leq a ; a)=\frac{E^{*} \delta}{\pi}\left\{\frac{1-2 v}{r^{2}}\left(a-\sqrt{a^{2}-r^{2}}\right)-\frac{1}{\sqrt{a^{2}-r^{2}}}\right\}, \\
& \sigma_{\varphi \varphi}^{\mathrm{FP}}(r \leq a ; a)=-\frac{E^{*} \delta}{\pi}\left\{\frac{1-2 v}{r^{2}}\left(a-\sqrt{a^{2}-r^{2}}\right)+\frac{2 v}{\sqrt{a^{2}-r^{2}}}\right\}, \\
& \sigma_{z z}^{\mathrm{FP}}(r \leq a ; a)=-\frac{E^{*} \delta}{\pi \sqrt{a^{2}-r^{2}}} .
\end{aligned}
$$

The last expression naturally reproduces the pressure distribution under the rigid cylindrical flat punch given by Boussinesq [1].

With the stress components beneath the surface given by Huber, we can also calculate the gradient of the hydrostatic pressure

$$
\frac{\mathrm{d} p}{\mathrm{~d} z}=\frac{\mathrm{d}}{\mathrm{d} z}\left\{-\frac{1}{3}\left(\sigma_{r r}+\sigma_{\varphi \varphi}+\sigma_{z z}\right)\right\}
$$

for the indentation by a parabolic rigid body. In the contact plane $z=0$, the expression reads

$$
\frac{\mathrm{d} p^{\mathrm{H}}}{\mathrm{d} z}(r ; a)=\frac{4 E^{*}(1+v)}{3 \pi R}\left\{\frac{\pi}{2}-H(r-a)\left[\arccos \left(\frac{a}{r}\right)+\frac{a}{\sqrt{r^{2}-a^{2}}}\right]\right\},
$$

where $H(r-a)$ is the Heaviside step function. Thus, the pressure gradient for indentation by a rigid flat cylindrical punch can thus be expressed as

$$
\frac{\mathrm{d} p^{\mathrm{FP}}}{\mathrm{d} z}(r ; a)=\frac{2 E^{*}(1+v) \delta}{3 \pi a}\left\{\delta_{\text {Dirac }}(r-a)\left[\arccos \left(\frac{a}{r}\right)+\frac{a}{\sqrt{r^{2}-a^{2}}}\right]-H(r-a) \frac{a^{2}}{\left(r^{2}-a^{2}\right)^{3 / 2}}\right\},
$$

where $\delta_{\text {Dirac }}(r-a)$ denotes the Dirac delta function.

\section{2 | Axisymmetric solution}

Let us now consider the general axisymmetric case.

Let the indenting body have a convex smooth profile $f=f(r)$, in which case the relation between penetration depth and contact radius is given by a unique function

$$
\delta=g(a) .
$$

We can interpret the indentation process as a series of incremental flat punch indentations with increasing contact radii. Hence,

$$
\sigma_{i j}^{\mathrm{AS}}=\int \mathrm{d} \sigma_{i j}^{\mathrm{AS}}=\int_{0}^{a} \frac{\partial \sigma_{i j}^{\mathrm{AS}}}{\partial \delta} \frac{\mathrm{d} g}{\mathrm{~d} u} \mathrm{~d} u=\int_{0}^{a} \frac{\partial \sigma_{i j}^{\mathrm{FP}}}{\partial \delta} \frac{\mathrm{d} g}{\mathrm{~d} u} \mathrm{~d} u
$$


and from Equation (3) we obtain the stresses in the surface for the axisymmetric case (a prime denotes the derivative),

$$
\begin{aligned}
& \sigma_{z z}^{\mathrm{AS}}(r ; a)=-\frac{E^{*}}{\pi} \int_{r}^{a} \frac{g^{\prime}(u) \mathrm{d} u}{\sqrt{u^{2}-r^{2}}}, \\
& \sigma_{r r}^{\mathrm{AS}}(r ; a)=\sigma_{z z}^{\mathrm{AS}}(r ; a)+\frac{1-2 v}{2 \pi r^{2}} F_{N}(a)-\frac{E^{*}(1-2 v)}{\pi r^{2}} \int_{r}^{a} \sqrt{u^{2}-r^{2}} g^{\prime}(u) \mathrm{d} u, \\
& \sigma_{\varphi \varphi}^{\mathrm{AS}}(r ; a)=(1+2 v) \sigma_{z z}^{\mathrm{AS}}(r ; a)-\sigma_{r r}^{\mathrm{AS}}(r ; a) .
\end{aligned}
$$

Here, the first relation is, of course, the solution by Galin [13] and Sneddon [14] for the "pressure" distribution in the axisymmetric Boussinesq problem, and $F_{N}$ is the total normal force,

$$
F_{N}(a)=2 E^{*} \int_{0}^{a}(\delta-g(u)) \mathrm{d} u .
$$

With Equation (6), we also obtain expressions for the pressure gradient in the surface for the axisymmetric case as shown in Appendix A. The resulting expressions read

$$
\begin{aligned}
& \frac{\mathrm{d} p^{\mathrm{AS}}}{\mathrm{d} z}(r \leq a ; a)=\frac{2 E^{*}(1+v)}{3 \pi}\left\{\frac{g^{\prime}(0)}{r}+\int_{0}^{r} \frac{g^{\prime \prime}(u) \mathrm{d} u}{\sqrt{r^{2}-u^{2}}}\right\}, \\
& \frac{\mathrm{d} p^{\mathrm{AS}}}{\mathrm{d} z}(r>a ; a)=-\frac{2 E^{*}(1+v)}{3 \pi} \int_{0}^{a} \frac{u g^{\prime}(u) \mathrm{d} u}{\left(r^{2}-u^{2}\right)^{3 / 2}} .
\end{aligned}
$$

In the framework of the Method of Dimensionality Reduction [15], it is easily shown that the function $g$ can be obtained from the profile function $f$ via the Abel integral transformation

$$
g(x)=|x| \int_{0}^{|x|} \frac{f^{\prime}(r)}{\sqrt{x^{2}-r^{2}}} \mathrm{~d} r
$$

\section{3 | TANGENTIAL CONTACT IN THE CATTANEO-MINDLIN APPROXIMATION}

Let us now calculate the stress state and the pressure gradient in the surface due to surface loads in the form

$$
\sigma_{z z}=\sigma_{y z}=0, \sigma_{x z} \neq 0, \quad r<a .
$$

Thereby the stresses $\sigma_{x z}$ arise from arbitrary tangential loading in the Cattaneo-Mindlin approximation, that is to say, they can be written as a finite superposition of contact pressure distributions resulting from the frictionless normal indentation problem.

\section{1 | The flat punch solution}

Again, we start with the Hertzian case. The contact is globally sliding, i.e. the tangential loading can be written in the form

$$
\sigma_{x z}^{\mathrm{H}}(r \leq a ; a)=\frac{2 \mu E^{*}}{\pi R} \sqrt{a^{2}-r^{2}},
$$


with the coefficient of friction $\mu$. The resulting stresses in the surface were given by Hamilton \& Goodman [8] (note, that there is a factor $\pi$ missing in Equation (10) of their paper). Within the contact area the non-vanishing stresses are independent of the contact radius and given by

$$
\sigma_{y y}^{\mathrm{H}}(x, y ; r \leq a)=\frac{3 v}{4+v} \sigma_{x x}^{\mathrm{H}}(x, y ; r \leq a)=\frac{x}{y} \frac{3 v}{2-v} \sigma_{x y}^{\mathrm{H}}(x, y ; r \leq a)=-\frac{3 \mu E^{*}}{4 R} v x .
$$

Outside the contact area the stresses in the surface read

$$
\begin{aligned}
& \sigma_{x x}^{\mathrm{H}}(x, y ; r>a ; a)=-\frac{2 \mu E^{*}}{\pi R} \frac{x}{r^{4}}\left\{2\left(r^{2}+v y^{2}\right) F+v\left(3-4 \frac{x^{2}}{r^{2}}\right) H\right\}, \\
& \sigma_{y y}^{\mathrm{H}}(x, y ; r>a ; a)=-\frac{2 \mu E^{*}}{\pi R} \frac{v x}{r^{4}}\left\{2 x^{2} F+\left(1-4 \frac{y^{2}}{r^{2}}\right) H\right\}, \\
& \sigma_{x y}^{\mathrm{H}}(x, y ; r>a ; a)=-\frac{2 \mu E^{*}}{\pi R} \frac{y}{r^{4}}\left\{\left(r^{2}-2 v x^{2}\right) F+v\left(1-4 \frac{x^{2}}{r^{2}}\right) H\right\},
\end{aligned}
$$

with the short-cuts

$$
\begin{aligned}
& F:=-\frac{a}{2} \sqrt{r^{2}-a^{2}}+\frac{r^{2}}{2} \arctan \left(\frac{a}{\sqrt{r^{2}-a^{2}}}\right), \\
& H:=\frac{a}{2}\left(\sqrt{r^{2}-a^{2}}\right)^{3}-\frac{r^{4}}{4} \arctan \left(\frac{a}{\sqrt{r^{2}-a^{2}}}\right)-\frac{a r^{2}}{4} \sqrt{r^{2}-a^{2}} .
\end{aligned}
$$

Via differentiation with respect to $a$, we obtain the stresses for sliding of a flat punch, i.e. resulting from a tangential load

$$
\sigma_{x z}^{\mathrm{FP}}(r \leq a ; a)=\frac{\mu E^{*} \delta}{\pi \sqrt{a^{2}-r^{2}}}
$$

As the stresses inside the contact area are independent of the contact radius in the parabolic case, they vanish for the flat punch. Hence, the non-vanishing stresses resulting from the tangential load (18) are

$$
\begin{aligned}
& \sigma_{x x}^{\mathrm{FP}}(x, y ; r>a ; a)=-\frac{\mu E^{*} \delta}{\pi} \frac{x}{r^{4}}\left\{2\left(r^{2}+v y^{2}\right) \frac{a}{\sqrt{r^{2}-a^{2}}}-2 v\left(3-4 \frac{x^{2}}{r^{2}}\right) a \sqrt{r^{2}-a^{2}}\right\}, \\
& \sigma_{y y}^{\mathrm{FP}}(x, y ; r>a ; a)=-\frac{\mu E^{*} \delta}{\pi} \frac{v x}{r^{4}}\left\{2 x^{2} \frac{a}{\sqrt{r^{2}-a^{2}}}-2\left(1-4 \frac{y^{2}}{r^{2}}\right) a \sqrt{r^{2}-a^{2}}\right\}, \\
& \sigma_{x y}^{\mathrm{FP}}(x, y ; r>a ; a)=-\frac{\mu E^{*} \delta}{\pi} \frac{y}{r^{4}}\left\{\left(r^{2}-2 v x^{2}\right) \frac{a}{\sqrt{r^{2}-a^{2}}}-2 v\left(1-4 \frac{x^{2}}{r^{2}}\right) a \sqrt{r^{2}-a^{2}}\right\} .
\end{aligned}
$$

A tangentially loaded cylindrical flat punch can, within the assumptions of the Cattaneo-Mindlin approximation, either completely stick or completely slip, with no difference in the form (18) of the tangential traction. Hence, the result for the sticking punch is elementarily given by the substitution

$$
\mu E^{*} \delta \rightarrow G^{*} u_{x, 0},
$$

with the effective shear modulus $G^{*}=4 G /(2-v)\left(G\right.$ is the shear modulus) and the tangential rigid body displacement $u_{x, 0}$.

In order to calculate the pressure gradient for the tangentially loaded parabolic indenter in the surface, the stresses beneath the surface are needed. These stresses are given as imaginary parts of a complex function in Hamilton \& Goodman [8]. However, they were later formulated, more conveniently, in explicit form by Hamilton [9]. Again, in compact form, the resulting expression for the pressure gradient in the contact plane is

$$
\frac{\mathrm{d} p^{\mathrm{H}}}{\mathrm{d} z}(x, y ; a)=\frac{4 \mu E^{*}(1+v)}{3 \pi R} H(a-r) \frac{x}{\sqrt{a^{2}-r^{2}}} .
$$


The gradient vanishes outside of the contact area. Hence, the pressure gradient in the surface in case of a sliding rigid flat cylindrical punch is

$$
\frac{\mathrm{d} p^{\mathrm{FP}}}{\mathrm{d} z}(x, y ; a)=\frac{2 \mu E^{*}(1+v) \delta}{3 \pi a}\left\{\delta_{\text {Dirac }}(a-r) \frac{x}{\sqrt{a^{2}-r^{2}}}-H(a-r) \frac{a x}{\left(a^{2}-r^{2}\right)^{3 / 2}}\right\} .
$$

The result for a sticking flat punch is readily obtained with the substitution (20).

\section{2 | Axisymmetric solution for monotonic tangential loading}

Let us consider an arbitrary axisymmetric sliding contact with a convex smooth profile. The contact configuration can be determined from a superposition of incremental sliding flat punch contacts with increasing radii. Hence, the stresses in the surface, resulting only from the tangential loading, are given by (for brevity we omit the arguments of the stresses)

$$
\begin{aligned}
& \sigma_{x x}^{\mathrm{AS}}=-\frac{\mu E^{*} x}{\pi r^{4}}\left\{2\left(r^{2}+v y^{2}\right) \int_{0}^{\min (r, a)} \frac{u g^{\prime}(u) \mathrm{d} u}{\sqrt{r^{2}-u^{2}}}-2 v\left(3-4 \frac{x^{2}}{r^{2}}\right) \int_{0}^{\min (r, a)} u g^{\prime}(u) \sqrt{r^{2}-u^{2}} \mathrm{~d} u\right\}, \\
& \sigma_{y y}^{\mathrm{AS}}=-\frac{\mu E^{*}}{\pi} \frac{v x}{r^{4}}\left\{2 x^{2} \int_{0}^{\min (r, a)} \frac{u g^{\prime}(u) \mathrm{d} u}{\sqrt{r^{2}-u^{2}}}-2\left(1-4 \frac{y^{2}}{r^{2}}\right) \int_{0}^{\min (r, a)} u g^{\prime}(u) \sqrt{r^{2}-u^{2}} \mathrm{~d} u\right\}, \\
& \sigma_{x y}^{\mathrm{AS}}=-\frac{\mu E^{*}}{\pi} \frac{y}{r^{4}}\left\{\left(r^{2}-2 v x^{2}\right) \int_{0}^{\min (r, a)} \frac{u g^{\prime}(u) \mathrm{d} u}{\sqrt{r^{2}-u^{2}}}-2 v\left(1-4 \frac{x^{2}}{r^{2}}\right) \int_{0}^{\min (r, a)} u g^{\prime}(u) \sqrt{r^{2}-u^{2}} \mathrm{~d} u\right\} .
\end{aligned}
$$

For the full stress state, the stresses (9) resulting from normal loading must be added.

In the case of monotonic tangential loading (constant normal force, increasing tangential force), slip will propagate from the edge of contact starting with the tangential loading. Due to the theorem by Jäger [16] and Ciavarella [17] the stresses $\sigma_{x z}$ (note that we operate within the Cattaneo-Mindlin approximation) are a superposition

$$
\sigma_{x z}^{\mathrm{AS}}(r ; a)=-\mu\left[\sigma_{z z}^{\mathrm{AS}}(r ; a)-\sigma_{z z}^{\mathrm{AS}}(r ; c)\right]
$$

with the radius $c$ of the stick area. Hence, the stresses in the surface resulting from the tangential loading in the partial slip case are given by

$$
\begin{aligned}
& \sigma_{x x}^{\mathrm{AS}}=-\frac{\mu E^{*} x}{\pi r^{4}}\left\{2\left(r^{2}+v y^{2}\right) \int_{\min (r, c)}^{\min (r, a)} \frac{u g^{\prime}(u) \mathrm{d} u}{\sqrt{r^{2}-u^{2}}}-2 v\left(3-4 \frac{x^{2}}{r^{2}}\right) \int_{\min (r, c)}^{\min (r, a)} u g^{\prime}(u) \sqrt{r^{2}-u^{2}} \mathrm{~d} u\right\}, \\
& \sigma_{y y}^{\mathrm{AS}}=-\frac{\mu E^{*}}{\pi} \frac{v x}{r^{4}}\left\{2 x^{2} \int_{\min (r, c)}^{\min (r, a)} \frac{u g^{\prime}(u) \mathrm{d} u}{\sqrt{r^{2}-u^{2}}}-2\left(1-4 \frac{y^{2}}{r^{2}}\right) \int_{\min (r, c)}^{\min (r, a)} u g^{\prime}(u) \sqrt{r^{2}-u^{2}} \mathrm{~d} u\right\}, \\
& \sigma_{x y}^{\mathrm{AS}}=-\frac{\mu E^{*}}{\pi} \frac{y}{r^{4}}\left\{\left(r^{2}-2 v x^{2}\right) \int_{\min (r, c)}^{\min (r, a)} \frac{u g^{\prime}(u) \mathrm{d} u}{\sqrt{r^{2}-u^{2}}}-2 v\left(1-4 \frac{x^{2}}{r^{2}}\right) \int_{\min (r, c)}^{\min (r, a)} u g^{\prime}(u) \sqrt{r^{2}-u^{2}} \mathrm{~d} u\right\} .
\end{aligned}
$$

Note that these stresses always vanish in the stick area. 
F I G U R E 1 Rigid conical indenter pressed into an elastic half-space

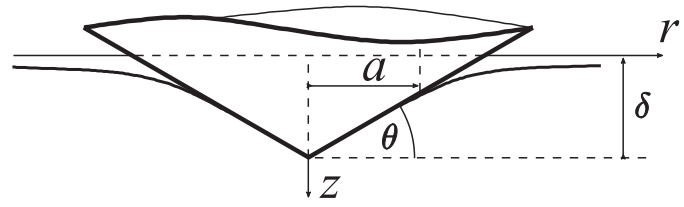

With Equation (22), we also obtain expressions for the pressure gradient in the surface for the tangentially loaded axisymmetric contact as shown in Appendix B. Considering an inner stick area, the expressions read

$$
\begin{gathered}
\frac{\mathrm{d} p^{\mathrm{AS}}}{\mathrm{d} z}(x, y ; r<c ; a)=\frac{2 \mu E^{*}(1+v) x}{3 \pi r^{2}}\left\{\frac{a g^{\prime}(a)}{\sqrt{a^{2}-r^{2}}}-\frac{c g^{\prime}(c)}{\sqrt{c^{2}-r^{2}}}-\int_{c}^{a} \frac{u g^{\prime \prime}(u) \mathrm{d} u}{\sqrt{u^{2}-r^{2}}}\right\}, \\
\frac{\mathrm{d} p^{\mathrm{AS}}}{\mathrm{d} z}(x, y ; c \leq r \leq a ; a)=\frac{2 \mu E^{*}(1+v) x}{3 \pi r^{2}}\left\{\frac{a g^{\prime}(a)}{\sqrt{a^{2}-r^{2}}}-\int_{r}^{a} \frac{u g^{\prime \prime}(u) \mathrm{d} u}{\sqrt{u^{2}-r^{2}}}\right\} .
\end{gathered}
$$

The pressure gradient always vanishes outside the contact area and the results for gross slip are readily obtained setting $c=0$.

\section{3 | Axisymmetric solution for the stress state for arbitrary loading histories}

For arbitrary loading histories the tangential loading is a finite superposition of the basic function (24) for monotonic loading [18]. The correct superposition, depending on the loading history, is most conveniently calculated with the method of dimensionality reduction (MDR, see for details [19], Chapter 4),

$$
\sigma_{x z}^{\mathrm{AS}}(r ; a)=-\frac{G^{*}}{\pi} \int_{r}^{a} \frac{\mathrm{d}}{\mathrm{d} u}\left[u_{x, 1 \mathrm{D}}(u)\right] \frac{\mathrm{d} u}{\sqrt{u^{2}-r^{2}}},
$$

with the one-dimensional tangential spring displacements $u_{x, 1 \mathrm{D}}$ in the MDR model. Hence, with (20), the stresses in the surface resulting from arbitrary tangential loading are given by

$$
\begin{aligned}
& \sigma_{x x}^{\mathrm{AS}}=\frac{G^{*} x}{\pi r^{4}} \int_{0}^{r} \frac{\mathrm{d}}{\mathrm{d} u}\left[u_{x, 1 \mathrm{D}}(u)\right]\left[\frac{2\left(r^{2}+v y^{2}\right)}{\sqrt{r^{2}-u^{2}}}-2 v\left(3-4 \frac{x^{2}}{r^{2}}\right) \sqrt{r^{2}-u^{2}}\right] u \mathrm{~d} u, \\
& \sigma_{y y}^{\mathrm{AS}}=\frac{G^{*} v x}{\pi r^{4}} \int_{0}^{r} \frac{\mathrm{d}}{\mathrm{d} u}\left[u_{x, 1 \mathrm{D}}(u)\right]\left[\frac{2 x^{2}}{\sqrt{r^{2}-u^{2}}}-2\left(1-4 \frac{y^{2}}{r^{2}}\right) \sqrt{r^{2}-u^{2}}\right] u \mathrm{~d} u, \\
& \sigma_{x y}^{\mathrm{AS}}=\frac{G^{*} y}{\pi r^{4}} \int_{0}^{r} \frac{\mathrm{d}}{\mathrm{d} u}\left[u_{x, 1 \mathrm{D}}(u)\right]\left[\frac{\left(r^{2}-2 v x^{2}\right)}{\sqrt{r^{2}-u^{2}}}-2 v\left(1-4 \frac{x^{2}}{r^{2}}\right) \sqrt{r^{2}-u^{2}}\right] u \mathrm{~d} u .
\end{aligned}
$$

For arbitrary loading, the function $u_{x, 1 \mathrm{D}}$ can only be obtained numerically.

\section{I EXAMPLE: CONICAL CONTACT}

A conical contact serves as a simple example for the application of the expressions for the general axisymmetric contact that were derived above. We consider a rigid cone with the profile $f(r)=r \tan \theta$ that is pressed into an elastic half-space as shown in Figure 1. The relation between penetration depth and contact radius for a cone has been found by Love [20],

$$
\delta=g(a)=\frac{\pi}{2} a \tan \theta .
$$



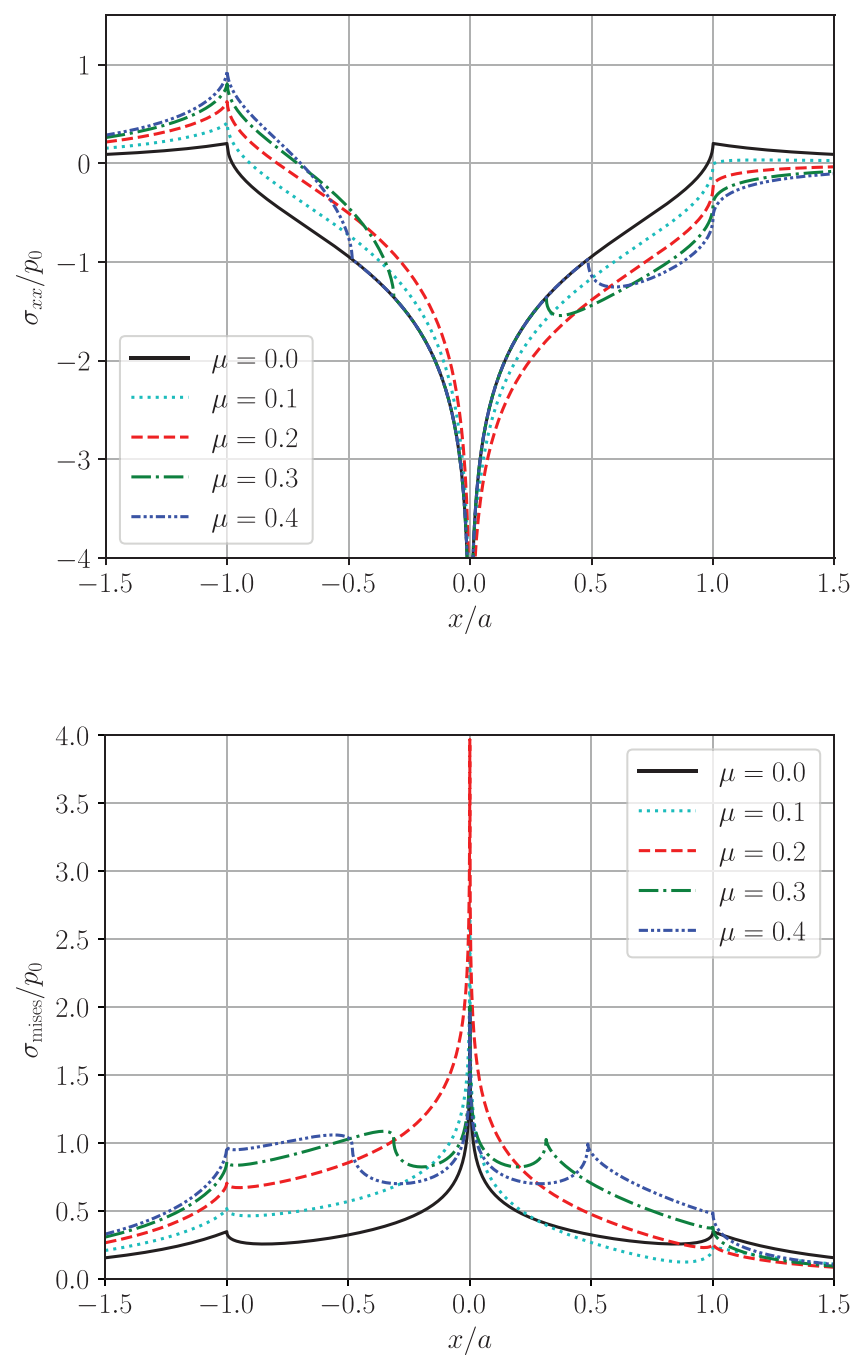

F I G U R E 2 Normalized stress $\sigma_{x x}$ at the surface of the half-space in the plane $y=0$ for a conical contact with indentation depth $\delta$ and tangential displacement $u_{x, 0}=\delta / 4$ as a function of the normalized $x$-coordinate for different friction coefficients $\mu$
F I G URE 3 Normalized von Mises equivalent stress $\sigma_{\text {mises }}$ at the surface of the half-space in the plane $y=0$ for a conical contact with indentation depth $\delta$ and tangential displacement $u_{x, 0}=\delta / 4$ as a function of the normalized $x$-coordinate for different friction coefficients $\mu$

With this relation, basically the contact problem is solved. Inserting the function $g$ into the expressions derived above, the solution for the stress components and the pressure gradient can be obtained, as given in Appendix C.

In Figure 2, the normalized stress $\sigma_{x x}$ for a conical contact with indentation depth $\delta$ and tangential displacement $u_{x, 0}=\delta / 4$ is plotted for different friction coefficients $\mu$. For all cases, $\nu=0.3$ is used. In the cases $\mu=0.3$ and $\mu=0.4$, the inner area of the indenter is sticking. Here, the stress $\sigma_{x x}$ is equal to the case without tangential loading $(\mu=0)$. For $\mu=0.1$ and $\mu=0.2$, the indenter is under gross slip.

As an often-used measure for the prediction of yielding, the von Mises equivalent stress has calculated as well. It is the square root of the second invariant of the stress deviator tensor. Hence,

$$
\sigma_{\text {mises }}^{2}=J_{2}=\frac{1}{2}\left\{\left(\sigma_{x x}-\sigma_{y y}\right)^{2}+\left(\sigma_{y y}-\sigma_{z z}\right)^{2}+\left(\sigma_{z z}-\sigma_{x x}\right)^{2}+6\left(\sigma_{x y}^{2}+\sigma_{x z}^{2}+\sigma_{y z}^{2}\right)\right\} .
$$

In Figure 3 and Figure 4 the normalized von Mises stress and the maximal and minimal principal stresses are shown in the line with $y=z=0$ for fixed normal and tangential displacements $u_{x, 0}=\delta / 2$ and different values of the friction coefficient. Figure 5 visualizes the respective relations for the pressure gradient.

\section{5 | DISCUSSION}

The concept of superposition of solutions for the rigid cylindrical flat punch is, of course, also applicable for the stress state and pressure gradient beneath the surface. Starting with the respective full solutions for the Hertzian problem the solutions for the axisymmetric case can be constructed without problems in the same fashion as above. Thus, the problem is again reduced to the 
F I G U R E 4 Normalized minimal and maximal principal stresses at the surface of the half-space in the plane $y=0$ for a conical contact with indentation depth $\delta$ and tangential displacement $u_{x, 0}=\delta / 4$ as a function of the normalized $x$-coordinate for different friction coefficients $\mu$

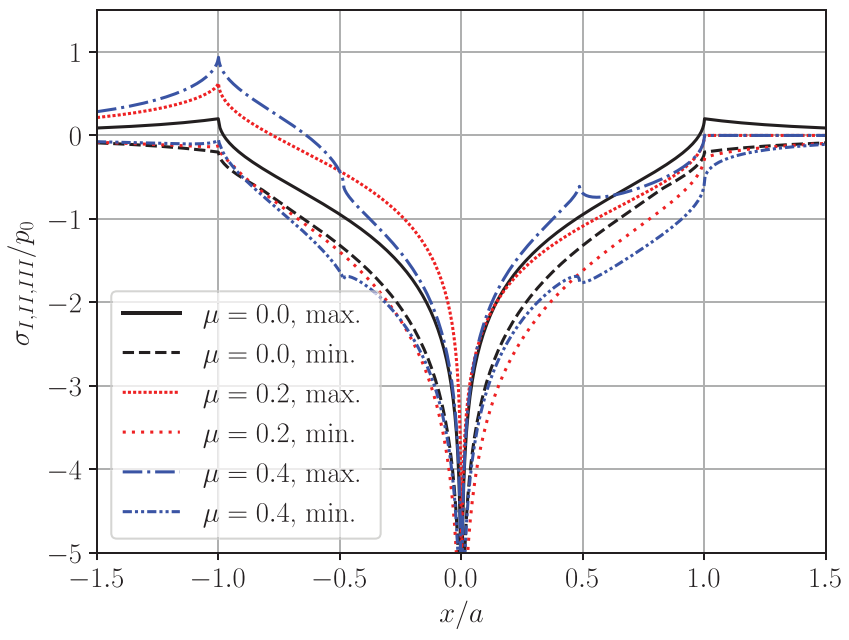

F I G U R E 5 Normalized gradient of the hydrostatic pressure at the surface of the half-space in the plane $y=0$ for a conical contact with indentation depth $\delta$ and tangential displacement $u_{x, 0}=\delta / 4$ as a function of the normalized $x$-coordinate for different friction coefficients $\mu$

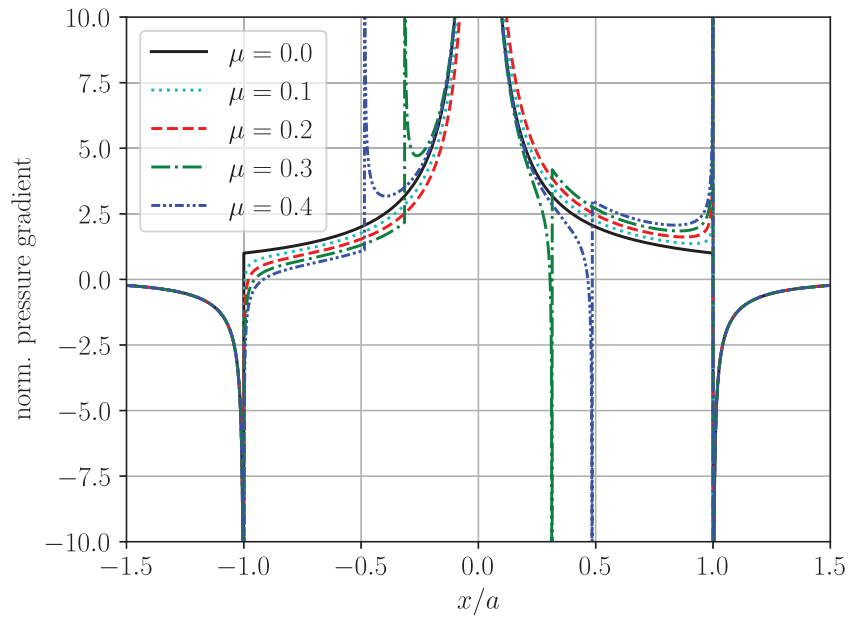

determination of one-dimensional integrals. However, we omit the general expressions here for brevity. Moreover, note that the validity of the superposition is only bound to the linearity of the problem. It is, for example, also valid for contact problems of layered [21] or functionally graded media [22].

The equations characterizing the state of stress in a contact, presented in the present paper, can be of interest, above all, for practical engineers. We provided the full stress tensor in the contact plane thus going over the textbook equations for only normal and tangential stress components in the surface. For the first time, the expressions are provided for an arbitrary axisymmetric profile with a compact contact area thus giving the possibility of analyzing arbitrary processes of shape changes, e.g. due to wear or (in natural joints) due to tissue growth. The full stress tensor gives much more detailed information about the stress state than the usually used normal and tangential in-plane stress components. They allow immediate determination of principal stresses and the maximum tangential stress and thus identification of the endangered regions with respect to plastic failure or fatigue cracking.

For natural biological joints, it was found experimentally, that the appearance of a pressure gradient in tissues and resulting fluid flow leads to the transport of macromolecular cytokines, growth factors, enzymes, and other substances and thus intensifies the processes of tissue growth [23]. For analyzing these processes, also the pressure gradient in the contact surface is of interest. It was derived in a general form. In the present paper, we limited ourselves to the presentation of the normal component $\partial p / \partial z$.

\section{6 | CONCLUSIONS}

Using the superposition idea by Mossakovski [11] and Jäger [24], we derived equations for the full stress tensor and the normal component of hydrostatic pressure gradient in the contact plane in the form of one-dimensional integrals. For the classical shapes as parabolic or conical profile, all integrals can be evaluated analytically. Nevertheless, the most important point is that 
the stresses and pressure gradients are provided also for arbitrary axisymmetric profiles with compact contact area. This allows for fast numerical simulations of contact problems involving continuous change of shape (e.g. wear or growth).

\section{ADDITIONAL INFORMATION}

\section{ACKNOWLEDGEMENTS}

The authors are very grateful to Justus Benad for valuable discussions on the topic.

\section{COMPETING INTERESTS}

None.

\section{AUTHOR CONTRIBUTIONS}

All authors contributed to the manuscript. EW and FF contributed analytical results, FF performed the numerical calculations. The manuscript was prepared by all authors.

\section{DATA AVAILABILITY STATEMENT}

No additional data (other than stated in the manuscript) was produced or used for the preparation of the manuscript.

\section{ORCID}

Emanuel Willert (D) https://orcid.org/0000-0001-7535-7301

\section{REFERENCES}

[1] J. Boussinesq, Applications des Potentiels a L'etude de L'equilibre et du Mouvement des Solides Elastiques. Gauthiers-Villars, Paris (in French) 1885.

[2] V. Cerruti, Ricerche intorno all'equilibrio de' corpi elastici isotropi, Rendiconte della Accademia Nazionale dei Lincei. 1882, 3, 81 (in Italian).

[3] J. R Barber, Contact Mechanics. Springer International Publishing AG, Cham. 2018

[4] H. Hertz, Über die Berührung fester elastischer Körper, Journal für die reine und angewandte Mathematik 1882, 92 , 156 (in German).

[5] M. T Huber, Zur Theorie der Berührung fester elastischer Körper, Ann. Phys. Lpz. 1904, 14, 153 (in German).

[6] A. Sackfield, D. A Hills, Some Useful Results in the Classical Hertz Contact Problem, The Journal of Strain Analysis for Engineering Design 1983, $18,101$.

[7] D. A. Hills, A. Sackfield, The Stress Field Induced by Normal Contact between Dissimilar Spheres, J. Appl. Mech. 1987, $54,8$.

[8] G. M. Hamilton, L. E Goodman, The Stress Field Created by a Circular Sliding Contact, J. Appl. Mech. 1966, $33,371$.

[9] G. M Hamilton, Explicit Equations for the Stresses beneath a Sliding Spherical Contact, Proc. Inst. Mech. Eng. Part C J. Mech. Eng. Sci. 1983, 197, 53.

[10] A. Sackfield, D. A Hills, Some Useful Results in the Tangentially Loaded Hertzian Contact Problem, The Journal of Strain Analysis for Engineering Design 1983, 18, 107.

[11] V. I Mossakovski, Compression of Elastic Bodies under Conditions of Adhesion (Axisymmetric Case), PMM Journal of Applied Mathematics and Mechanics 1963, 27, 630.

[12] M. Heß, Über die exakte Abbildung ausgewählter dreidimensionaler Kontakte auf Systeme mit niedrigerer räumlicher Dimension. CuvillierVerlag, Göttingen (in German) 2011.

[13] L. A Galin, Three-Dimensional Contact Problems of the Theory of Elasticity for Punches with a Circular Planform, Prikl. Mat. Mekh. 1946, 10, 425 (in Russian).

[14] I. N Sneddon, The relation between load and penetration in the axisymmetric Boussinesq problem for a punch of arbitrary profile, Int. J. Eng. Sci. 1965, 3, 47.

[15] V. L. Popov, M. Heß, Method of Dimensionality Reduction in Contact Mechanics and Friction. Springer Verlag, Berlin 2015.

[16] J. Jäger, A New Principle in Contact Mechanics, J. Tribol. 1998, 120, 677.

[17] M. Ciavarella, Tangential Loading of General Three-Dimensional Contacts, J. Appl. Mech. 1998, 65, 998.

[18] J. Jäger, Elastic contact of equal spheres under oblique forces, Arch. Appl. Mech. (Germany) 1993, 63, 402.

[19] V. L. Popov, M. Heß, E. Willert, Handbook of Contact Mechanics: Exact Solutions of Axisymmetric Contact Problems. Springer Verlag, Berlin 2019.

[20] A. E. H Love, Boussinesq's Problem for a Rigid Cone, The Quarterly Journal of Mathematics 1939, $10,161$. 
[21] I. I. Argatov, M. Heß, V. L Popov, The extension of the method of dimensionality reduction to layered elastic media, ZAMM Z. Angew. Math. Mech. (Germany) 2018, 98, 622.

[22] M. Heß, A simple method for solving adhesive and non-adhesive axisymmetric contact problems of elastically graded materials, Int. J. Eng. Sci. 2016, 104, 20.

[23] A. J. Grodzinsky, M. E. Levenston, M. Jin, E. H Frank, Cartilage Tissue Remodeling in Response to Mechanical Forces, Annu. Rev. Biomed. Eng. 2000, 2, 691

[24] J. Jäger, Axi-symmetric bodies of equal material in contact under torsion or shift, Arch. Appl. Mech. (Germany) 1995, 65, 478.

How to cite this article: E. Willert, F. Forsbach, V. L. Popov. Stress tensor and gradient of hydrostatic pressure in the contact plane of axisymmetric bodies under normal and tangential loading. Z Angew Math Mech. 2020;100:e201900223. https://doi.org/10.1002/zamm.201900223

\section{APPENDIX A: DERIVATION OF THE PRESSURE GRADIENT FOR THE NORMAL CONTACT PROBLEM}

Using Equations (2) and (6), the gradient of the hydrostatic pressure for the axisymmetric contact is

$$
\frac{\mathrm{d} p^{\mathrm{AS}}}{\mathrm{d} z}(r ; a)=C \int_{0}^{a}\left\{\delta_{\text {Dirac }}(r-u)\left[\frac{1}{u} \arccos \left(\frac{u}{r}\right)+\frac{1}{\sqrt{r^{2}-u^{2}}}\right]-H(r-u) \frac{u}{\left(r^{2}-u^{2}\right)^{3 / 2}}\right\} g^{\prime}(u) \mathrm{d} u,
$$

where $C=\frac{2 E^{*}(1+v)}{3 \pi}$ Inside the contact this can be simplified to

$$
\begin{aligned}
& \frac{\mathrm{d} p^{\mathrm{AS}}}{\mathrm{d} z}(r<a ; a)=C\left\{\lim _{u \rightarrow r}\left(\frac{g^{\prime}(u)}{\sqrt{r^{2}-u^{2}}}\right)-\int_{0}^{r} \frac{u g^{\prime}(u)}{\left(r^{2}-u^{2}\right)^{3 / 2}} \mathrm{~d} u\right\} \\
& \Leftrightarrow \frac{\mathrm{d} p^{\mathrm{AS}}}{\mathrm{d} z}(r<a ; a)=C\left\{\lim _{u \rightarrow r}\left(\frac{g^{\prime}(u)}{\sqrt{r^{2}-u^{2}}}\right)-\lim _{u \rightarrow r}\left(\frac{g^{\prime}(u)}{\sqrt{r^{2}-u^{2}}}\right)+\frac{g^{\prime}(0)}{r}+\int_{0}^{r} \frac{g^{\prime \prime}(u)}{\sqrt{r^{2}-u^{2}}} \mathrm{~d} u\right\} \\
& \Leftrightarrow \frac{\mathrm{d} p^{\mathrm{AS}}}{\mathrm{d} z}(r<a ; a)=C\left\{\frac{g^{\prime}(0)}{r}+\int_{0}^{r} \frac{g^{\prime \prime}(u)}{\sqrt{r^{2}-u^{2}}} \mathrm{~d} u\right\},
\end{aligned}
$$

where the limits are understood in the sense of Cauchy principal values to cancel them out. Outside the contact area, the only non-vanishing term in Equation (A.1) is

$$
\frac{\mathrm{d} p^{\mathrm{AS}}}{\mathrm{d} z}(r>a ; a)=-C \int_{0}^{r} \frac{u g^{\prime}(u)}{\left(r^{2}-u^{2}\right)^{3 / 2}} \mathrm{~d} u .
$$

\section{APPENDIX B: DERIVATION OF THE PRESSURE GRADIENT FOR THE TANGENTIAL CONTACT PROBLEM}

Using Equations (2) and (22), the gradient of the hydrostatic pressure for the axisymmetric contact resulting only from the tangential loading is

$$
\frac{\mathrm{d} p^{\mathrm{AS}}}{\mathrm{d} z}(r ; a)=\mu C \frac{x}{r^{2}} \int_{0}^{a}\left\{\delta_{\text {Dirac }}(u-r) \frac{r^{2}}{u \sqrt{u^{2}-r^{2}}}-H(u-r) \frac{r^{2}}{\left(u^{2}-r^{2}\right)^{3 / 2}}\right\} g^{\prime}(u) \mathrm{d} u .
$$


Hence, it vanishes outside of the contact area. Inside the contact area, we can simplify the expression to

$$
\begin{aligned}
& \frac{\mathrm{d} p^{\mathrm{AS}}}{\mathrm{d} z}(r<a ; a)=\mu C \frac{x}{r^{2}}\left\{\lim _{u \rightarrow r}\left(\frac{r^{2} g^{\prime}(u)}{u \sqrt{u^{2}-r^{2}}}\right)-\int_{r}^{a} \frac{r^{2} g^{\prime}(u)}{\left(u^{2}-r^{2}\right)^{3 / 2}} \mathrm{~d} u\right\} \\
& \Leftrightarrow \frac{\mathrm{d} p^{\mathrm{AS}}}{\mathrm{d} z}(r<a ; a)=\mu C \frac{x}{r^{2}}\left\{\lim _{u \rightarrow r}\left(\frac{r^{2} g^{\prime}(u)}{u \sqrt{u^{2}-r^{2}}}\right)+\frac{a g^{\prime}(a)}{\sqrt{a^{2}-r^{2}}}-\lim _{u \rightarrow r}\left(\frac{u g^{\prime}(u)}{\sqrt{u^{2}-r^{2}}}\right)-\int_{r}^{a} \frac{u g^{\prime \prime}(u)}{\sqrt{u^{2}-r^{2}}} \mathrm{~d} u\right\} \\
& \Leftrightarrow \frac{\mathrm{d} p^{\mathrm{AS}}}{\mathrm{d} z}(r<a ; a)=\mu C \frac{x}{r^{2}}\left\{\frac{a g^{\prime}(a)}{\sqrt{a^{2}-r^{2}}}-\int_{r}^{a} \frac{u g^{\prime \prime}(u)}{\sqrt{u^{2}-r^{2}}} \mathrm{~d} u\right\},
\end{aligned}
$$

where the limits again are understood as Cauchy principal values.

\section{APPENDIX C: SOLUTION FOR STRESS COMPONENTS AND PRESSURE GRADIENT IN CONICAL CONTACT}

Using Equations (9), the non-vanishing stress components of the normal contact problem are

$$
\begin{aligned}
& \sigma_{z z}^{\mathrm{CN}}(r<a ; a)=-p_{0} \ln \left(\frac{a}{r}+\sqrt{\frac{a^{2}}{r^{2}}-1}\right), \\
& \sigma_{r r}^{\mathrm{CN}}(r<a ; a)=-p_{0}\left\{\ln \left(\frac{a}{r}+\sqrt{\frac{a^{2}}{r^{2}}-1}\right)-\frac{(1-2 v)}{2}\left[\frac{a^{2}}{r^{2}}\left(1-\sqrt{1-\frac{r^{2}}{a^{2}}}\right)-\ln \left(\frac{r}{a+\sqrt{a^{2}-r^{2}}}\right)\right]\right\}, \\
& \sigma_{r r}^{\mathrm{CN}}(r>a ; a)=p_{0} \frac{(1-2 v) a^{2}}{2 r^{2}}, \\
& \sigma_{\varphi \varphi}^{\mathrm{CN}}(r<a ; a)=-p_{0}\left\{2 v \ln \left(\frac{a}{r}+\sqrt{\frac{a^{2}}{r^{2}}-1}\right)+\frac{(1-2 v)}{2}\left[\frac{a^{2}}{r^{2}}\left(1-\sqrt{1-\frac{r^{2}}{a^{2}}}\right)-\ln \left(\frac{r}{a+\sqrt{a^{2}-r^{2}}}\right)\right]\right\}, \\
& \sigma_{\varphi \varphi}^{\mathrm{CN}}(r>a ; a)=-p_{0} \frac{(1-2 v) a^{2}}{2 r^{2}},
\end{aligned}
$$

with $p_{0}=E^{*} \tan \theta / 2$. Using Equations (11), the pressure gradient of the normal contact problem is

$$
\begin{aligned}
& \frac{\mathrm{d} p^{\mathrm{CN}}}{\mathrm{d} z}(r<a ; a)=p_{0} \frac{2(1+v)}{3} \frac{1}{r}, \\
& \frac{\mathrm{d} p^{\mathrm{CN}}}{\mathrm{d} z}(r>a ; a)=p_{0} \frac{2(1+v)}{3}\left(\frac{1}{r}-\frac{1}{\sqrt{r^{2}-a^{2}}}\right) .
\end{aligned}
$$

If the cone is displaced tangentially by $u_{x, 0}$, partial or gross slip will occur. In the case of partial slip, the radius of the inner stick area $c$ is determined by the condition [19], (Equation (4.27))

$$
G^{*} u_{x, 0}=\mu E^{*}(\delta-g(c)) \Rightarrow c=a-\frac{G^{*} u_{x, 0}}{\mu E^{*} \pi \tan \theta} .
$$


Using Equations (24) and (25), the non-vanishing stress components of the tangential contact problem are

$$
\begin{aligned}
\sigma_{x z}^{\mathrm{CT}}(r<c ; a) & =\mu p_{0}\left\{\ln \left(\frac{a}{r}+\sqrt{\frac{a^{2}}{r^{2}}-1}\right)-\ln \left(\frac{c}{r}+\sqrt{\frac{c^{2}}{r^{2}}-1}\right)\right\}, \\
\sigma_{x z}^{\mathrm{CT}}(c<r<a ; a) & =\mu p_{0} \ln \left(\frac{a}{r}+\sqrt{\frac{a^{2}}{r^{2}}-1}\right), \\
\sigma_{x x}^{\mathrm{CT}}(c<r<a ; a) & =-\mu p_{0} \frac{x}{r}\left\{2\left(1+v \frac{y^{2}}{r^{2}}\right) C-\frac{2 v}{3}\left(3-4 \frac{x^{2}}{r^{2}}\right) C^{3}\right\}, \\
\sigma_{x x}^{\mathrm{CT}}(r>a ; a) & =-\mu p_{0} \frac{x}{r}\left\{2\left(1+v \frac{y^{2}}{r^{2}}\right)(C-A)-\frac{2 v}{3}\left(3-4 \frac{x^{2}}{r^{2}}\right)\left(C^{3}-A^{3}\right)\right\}, \\
\sigma_{y y}^{\mathrm{CT}}(c<r<a ; a) & =-\mu p_{0} v \frac{x}{r}\left\{2 \frac{x^{2}}{r^{2}} C-\frac{2}{3}\left(1-4 \frac{y^{2}}{r^{2}}\right) C^{3}\right\}, \\
\sigma_{y y}^{\mathrm{CT}}(r>a ; a) & =-\mu p_{0} v \frac{x}{r}\left\{2 \frac{x^{2}}{r^{2}}(C-A)-\frac{2}{3}\left(1-4 \frac{y^{2}}{r^{2}}\right)\left(C^{3}-A^{3}\right)\right\}, \\
\sigma_{x y}^{\mathrm{CT}}(c<r<a ; a) & =-\mu p_{0} \frac{y}{r}\left\{\left(1-2 v \frac{x^{2}}{r^{2}}\right) C-\frac{2 v}{3}\left(1-4 \frac{x^{2}}{r^{2}}\right) C^{3}\right\}, \\
\sigma_{x y}^{\mathrm{CT}}(r>a ; a) & =-\mu p_{0} \frac{y}{r}\left\{\left(1-2 v \frac{x^{2}}{r^{2}}\right)(C-A)-\frac{2 v}{3}\left(1-4 \frac{x^{2}}{r^{2}}\right)\left(C^{3}-A^{3}\right)\right\},
\end{aligned}
$$

with the shortcuts

$$
A=\sqrt{1-\frac{a^{2}}{r^{2}}} \text { and } C=\sqrt{1-\frac{c^{2}}{r^{2}}} .
$$

With Equations (26), the pressure gradient for the tangential contact is

$$
\begin{aligned}
& \frac{\mathrm{d} p}{\mathrm{~d} z} \quad(r<c ; a)=\mu p_{0} \frac{2(1+v)}{3} \frac{x}{r^{2}}\left(\frac{a}{\sqrt{a^{2}-r^{2}}}-\frac{c}{\sqrt{c^{2}-r^{2}}}\right), \\
& \frac{\mathrm{d} p}{\mathrm{~d} z}^{\mathrm{CT}}(c<r<a ; a)=\mu p_{0} \frac{2(1+v)}{3} \frac{x a}{r^{2} \sqrt{a^{2}-r^{2}}} .
\end{aligned}
$$

In the case of gross slip, that is

$$
\frac{G^{*} u_{x, 0}}{\mu E^{*} \delta}>1
$$

one has to set $c=0$ in Equations (C.4) and (C.6). The full stress state and pressure gradient for the tangentially loaded contact is given by the superposition

$$
\sigma_{i j}=\sigma_{i j}{ }^{\mathrm{CN}}+\sigma_{i j}{ }^{\mathrm{CT}} \quad \text { and } \quad \frac{\mathrm{d} p}{\mathrm{~d} z}=\frac{\mathrm{d} p}{\mathrm{~d} z}^{\mathrm{CN}}+\frac{\mathrm{d} p}{\mathrm{~d} z}^{\mathrm{CT}} \text {. }
$$

